\title{
Transatlantica
}

Revue d'études américaines. American Studies Journal

1 | 2017

Morphing Bodies: Strategies of Embodiment in Contemporary US Cultural Practices

\section{"Things to be Forgotten": African American Family Histories, Silences, and the American Historical Profession in the Post-emancipation Era}

Conférence de Kendra Field, Assistant Professor en histoire et Africana Studies, Tufts University. Centre de Recherches sur l'Amérique du Nord (CRAN), Université Sorbonne Nouvelle - Paris III, 12 juin 2017.

Pierre Cras

\section{(Q) OpenEdition}

\section{Journals}

Édition électronique

URL : https://journals.openedition.org/transatlantica/8623

DOI : 10.4000/transatlantica.8623

ISSN : 1765-2766

Éditeur

Association française d'Etudes Américaines (AFEA)

Référence électronique

Pierre Cras, «"Things to be Forgotten": African American Family Histories, Silences, and the American Historical Profession in the Post-emancipation Era », Transatlantica [En ligne], 1 | 2017, mis en ligne le 03 décembre 2018, consulté le 22 mai 2021. URL : http://journals.openedition.org/transatlantica/8623 ; DOI : https://doi.org/10.4000/transatlantica.8623

Ce document a été généré automatiquement le 22 mai 2021

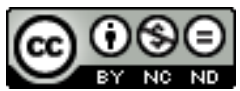

Transatlantica - Revue d'études américaines est mise à disposition selon les termes de la licence Creative Commons Attribution - Pas d'Utilisation Commerciale - Pas de Modification 4.0 International. 


\section{"Things to be Forgotten": African American Family Histories, Silences, and the American Historical Profession in the Post-emancipation}

\section{Era}

Conférence de Kendra Field, Assistant Professor en histoire et Africana Studies, Tufts University. Centre de Recherches sur l'Amérique du Nord (CRAN), Université Sorbonne Nouvelle - Paris III, 12 juin 2017.

Pierre Cras

1 L'intervention de Kendra Field s'inscrivait dans le cadre de ses propres travaux de recherche consacrés à la question de la mémoire familiale africaine-américaine, à ses modalités de transmission et ses nombreuses implications à la fois au sein de la sphère privée mais aussi à l'intérieur du monde universitaire. En effet, Kendra Field soutient que ses observations sur le sujet de l'histoire familiale - qui fut le point de départ de son ouvrage Growing Up with the Country : Family Race, and Nation After the Civil War doivent être (re)considérées à l'aune de l'historiographie et de la méthodologie de l'histoire africaine-américaine. Ainsi, le professeur Field a proposé entre autres une réflexion sur le statut de l'historien africain-américain et a voulu questionner les rapports de ce dernier aux deux notions $d^{\prime}$ « histoire » et de « mémoire ».

2 Kendra Field a commencé par expliquer sa démarche qui consiste à confronter la mémoire de sa propre famille et les archives qui confirment (ou infirment) les événements et dires relatifs au vécu de ses ancêtres. Elle a ainsi effectué une distinction nette entre la mémoire potentiellement remaniée lorsqu'elle se transmet d'un individu à l'autre, et l'histoire des familles telle qu'elle apparaît à travers diverses sources auxquelles les universitaires ont recours. Kendra Field a conclu ce préambule en expliquant son intérêt pour la récente augmentation du nombre de travaux universitaires, américains notamment, qui s'inscrivent dans le champ de 
l'historiographie familiale africaine-américaine. Elle a précisé finalement sa méthodologie et son choix de se pencher en priorité sur les écrits des intellectuels noirs américains W. E. B. Du Bois et Carter G. Woodson qui ont œuvré au processus de mémorialisation après la période de la Reconstruction (1865-1877).

La première partie de la conférence était dévolue à l'étude du parcours de W. E. B. Du Bois et à la façon dont ce dernier participa à la mise en place de la profession d'historien aux États-Unis. Citant un extrait tiré de son ouvrage, Black Reconstruction (1935), Kendra Field a rappelé à son auditoire qu'une écrasante partie de l'historiographie américaine de la première moitié du XXe siècle suivait une certaine posture intellectuelle teintée de l'idéologie raciste héritée de l'ère des lois Jim Crow. Dès lors qu'il s'agissait de retracer les avancées politiques apportées par la Reconstruction, nombre d'historiens s'affranchissaient volontairement du principe fondamental de l'objectivité, qu'ils sacrifiaient au nom d'une idéologie biaisée par leur patriotisme. Kendra Field a ensuite fait mention du contexte d'hostilité entre Du Bois et Woodson - le premier docteur en histoire dont les parents étaient nés esclaves aux États-Unis - d'un côté, et les nombreux partisans de William A. Dunning, le chantre de la « Dunning School» qui considérait la Reconstruction comme une « ère tragique de chaos orchestrée par les Noirs ", de l'autre. Les détracteurs de Du Bois et Woodson, majoritairement blancs, affirmaient qu'en raison de leur appartenance ethnique, ceuxci ne pouvaient prétendre à une analyse historique distanciée et objective de la période de la Reconstruction. Kendra Field a également évoqué la méfiance vis-à-vis d'un contre-discours académique qui ne se limitait pas aux travaux de Du Bois et Woodson mais concernait en réalité toutes les productions scientifiques des autres historiens professionnels africains-américains. Ces derniers ne pouvaient par ailleurs aspirer qu'à une diffusion restreinte de leurs recherches par le biais de publications très spécialisées telles le Journal of Negro History ou de monographies qui furent systématiquement déconsidérées par les historiens blancs entre les années 1910 et 1920.

Kendra Field précisa néanmoins que la décision d'exclure de facto les historiens professionnels africains-américains sur la base de leur manque d'objectivité ne faisait pas l'unanimité au sein du monde universitaire. Elle prit soin de mentionner la volonté connexe d'Albert Bushnell Hart - l'un des professeurs de Du Bois à Harvard - de considérer l'historiographie existante sous de nouveaux angles d'approches reposant sur l'exploitation minutieuse de sources originales.

5 De son côté, Du Bois n'eut de cesse de combattre le postulat raciste sur lequel reposait cette "controverse de l'objectivité ». A travers la publication de nombreux articles, notamment pour The Crisis, le journal de la NAACP, et de son ouvrage Black Reconstruction (1935) ou encore de sa participation à un grand nombre de manifestations pendant la période de la Renaissance de Harlem, Du Bois œuvrait en faveur d'un projet de publication monumentale intitulée Encylopedia of the Negro, qui devait regrouper les travaux des "meilleurs et des plus brillants » jeunes intellectuels africains-américains tels que Ralph Bunche, Rayford Logan ou Allison Davis, mais ne vit finalement jamais le jour en dépit des efforts incessants de Du Bois.

6 Les années 1930 virent finalement naître un nouveau courant universitaire qui remettait en perspective critique l'historiographie de l'esclavage et de la Reconstruction à l'aune de ses propres biais de classe et de race. Kendra Field a mentionné toutefois le fait que cette nouvelle école de pensée n'était pas le fait des historiens africains-américains, bien peu nombreux à avoir investi la profession depuis 
le premier article de Du Bois pour l'American Historical Review (1910). Ce furent le contexte de la Première Guerre mondiale et les transformations politiques d'aprèsguerre qui favorisèrent l'émergence d'un «relativisme historique » mettant à mal le discours normatif sur la profession d'historien.

7 La deuxième partie de la conférence est revenue sur les histoires familiales africainesaméricaines et l'appétence pour la généalogie après la période de l'émancipation (1863). Kendra Field associa ce besoin de connaissances du passé à la volonté personnelle des descendants d'esclaves de retracer non seulement l'histoire et l'arbre généalogique de leurs familles respectives, mais aussi de « bâtir l'histoire d'un peuple ». Elle affirma ensuite que les cinquante années qui suivirent l'émancipation marquèrent une première étape dans cette construction mémorielle à la fois collective et individuelle, à une époque où la «narration orale et / ou le storytelling » prévalaient sur toute autre forme de transmission du savoir.

8 Furent ensuite mentionnées les difficultés que Kendra Field a rencontrées ces dix dernières années, notamment à collecter des récits en histoire orale au sein desquels les femmes africaines-américaines jouaient invariablement un rôle mineur en comparaison de leurs homologues masculins, même si elles étaient les gardiennes de récits d'histoire familiale dont elles s'efforçaient de ne diffuser que les épisodes les moins tragiques et les plus respectables. Elle a alors cité l'exemple de sa grand-tante Marzetta Brown Wesley qui rédigea un court texte sur l'histoire de sa famille en 1985 et valorisa à cette occasion le parcours personnel d'un homme. Celle-ci se fit à cette occasion le relais du récit de vie de son propre grand-père Monroe Colman, qui était à la fois un homme d'affaires à succès et un pilier de la communauté.

9 Au gré de ses recherches et de sa collecte d'informations auprès de sa grand-mère et de ses grand-tantes, Kendra Field a mis au jour deux aspects qui sous-tendent ces omissions volontaires et dont les "non-dits" étaient en eux-mêmes révélateurs. D'abord, la prééminence des violences sexuelles subies par les Africaines-Américaines pendant la migration post-émancipation (après 1865). Les récits familiaux transmis par voie orale taisaient ces épisodes tragiques, mais il ne faisait nul doute que les actes de lynchage et de violence physique qui ont poussé les familles noires du Sud à migrer vers le Nord s'accompagnaient de la menace constante de viols. Ensuite, Kendra Field a établi un lien de corrélation entre les silences volontaires des récits familiaux et les chants d'esclaves. Pour illustrer ceci, elle convia l'une des membres de sa famille, présente à la conférence, à interpréter le célèbre gospel « Swing Low, Sweet Chariot ».

Cet intermède musical a servi de transition à Kendra Field qui a ensuite évoqué le parcours de l'enseignante et chanteuse africaine-américaine Ella Sheppard Moore qui déclarait en 1911 que " les chants des esclaves étaient intimement associés à la condition servile ainsi qu'à un sombre passé. Ces chants représentent ce qui sera inéluctablement oublié ». A travers son évocation de l'affiliation entre Ella Sheppard et l'Université noire de Fisk (Nashville, Tennessee), Kendra Field indiqua que la chanteuse et quelques autres étudiants en musique ont su s'émanciper de la pratique exclusive de la musique classique qui prévalait jusqu'alors à Fisk. Les chants religieux des esclaves, les "Jubilee Songs", passèrent ainsi de la sphère privée de quelques étudiants et professeurs de l'Université à une diffusion nationale portée par la chorale des «Fisk Jubilee Singers » à partir de 1872. Toutefois, Kendra Field précisa que cette pratique publique des chants d'esclaves restait relativement marginale et ambigüe. Les familles et individus de la génération post-émancipation après 1865 étaient en effet 
généralement désireux de ne pas laisser transparaître en public les séquelles de la période traumatique de l'esclavage que ces chants évoquaient. Une citation d'un ancien diplômé de Fisk, John Wesley Work II, vint confirmer cette volonté lorsqu'en en 1915 il affirmait dans son ouvrage Folk Songs of the American Negro: «lorsque les Noirs se sont retrouvés libres, ils ont littéralement mis leur passé derrière eux ». En ce sens, il était logique que les "Jubilee Songs" soient considérées comme une trace d'un passé douloureux qu'il leur fallait oublier, aussi mélodieuses soient-elles.

11 Dans les années 1890, la première génération de Noirs libres commença à se penser comme une " communauté » étendue et non plus comme un groupement d'individus séparés par l'Institution Particulière. Aux petites annonces publiées dans les journaux qui visaient à réunir les familles dispersées par l'esclavage, se superposèrent des éléments de langage faisant mention d'autres personnes de "leur peuple ", au début du XXe siècle. Kendra Field a souligné combien cette volonté de se percevoir en tant que membre d'un groupe ne se limitait plus à la cellule familiale stricto sensu et marquait un passage vers la construction d'une histoire commune. Elle a mentionné également le fait que ces événements s'inscrivaient dans le contexte d'un âge d'or du nationalisme noir américain combinant des revendications d'appartenance à un peuple / une " race " à une volonté de recherche d'une histoire commune qui rejetait le sentiment de honte éprouvé par les anciens esclaves, désormais âgés.

12 Les silences d'autrefois devinrent alors des éléments à part entière de l'histoire des familles noires aux États-Unis, comme en témoigne la publication en 1892 de l'ouvrage d'Anna Julia Cooper, A Voice from the South. Mais plus encore qu'une simple «tendance ", ce fait marquant s'est accompagné du développement de la presse noire dans les années 1880 qui joua un rôle fondamental dans la circulation d'informations et d'idées relatives à l'histoire et à la mémoire de l'esclavage au sein d'une communauté noire en devenir. Elle a de plus évoqué l'apport des auteurs et artistes qui participèrent de cette nouvelle dynamique commune à la fois au New Negro Movement d'Alain Locke, à la Renaissance de Harlem et aux premières années de la Grande Migration des Noirs du Sud vers les grandes villes du Nord. A l'instar du périodique The Crisis, de nombreux journaux africains-américains publièrent ainsi dans les années 1920 et 1930 des textes et des poèmes rendant hommage aux anciens esclaves comme celui de Charles Bertram «An Old Ex-Slave».

13 La conférencière a ensuite abordé un exemple antérieur aux années 1930 mais qui fut tout aussi significatif dans le processus de mémorialisation africain-américain, soit une cérémonie organisée le 1er janvier 1893 à Hampton en Virginie par une certaine Miss A. M. Bacon et qui s'intitulait «Exercices in Honor of the Aged : A Tribute to the Freedman from their Children». Cet événement amorçait en réalité un processus de reconnaissance officielle de l'histoire des anciens esclaves et montrait que la première génération d'Africains-Américains nés après l'esclavage intégrait à son récit mémoriel les expériences traumatiques de ceux «dont les rides sont les sillons de l'histoire américaine ", créant ainsi un lien entre passé, présent et futur. La question de la transmission mémorielle intervint également à travers les chansons et textes qui furent composés pour cette occasion puis publiés ensuite dans les pages du Southern Workman. Ce même journal servit à son tour de plateforme de diffusion pour Miss A.M. Bacon qui, en 1893, y fit paraitre un dossier spécial appelant à la constitution urgente de collections d'archives historiques et ethnologiques valorisant les contes, coutumes, traditions, proverbes, chants et musiques africains et africains-américains. Par cet 
appel, elle espérait marquer ainsi le commencement d'une vaste entreprise de préservation institutionnelle de la mémoire africaine et diasporique et elle incluait l'esclavage dans un continuum historique situé entre l'Afrique et les États-Unis.

Avant de conclure sa conférence, Kendra Field insista sur l'importance de la transmission mémorielle orale interpersonnelle et intergénérationnelle entre les anciens esclaves et leurs descendants, notamment grâce à la musique qui était une des premières «méthodes » de préservation d'un savoir historique selon Miss A. M. Bacon. Cette dernière suggéra également que les chants et musiques des esclaves - qui n'étaient pas encore conservés à l'échelle nationale - représentent " une chaîne dont les maillons relient les Africains-Américains aux Africains ». Ce furent par la suite les avancées culturelles et politiques apportées par le New Negro Movement qui nourrirent l'attrait pour l'histoire africaine-américaine et la diffusion de sa mémoire culturelle pendant l'entre-deux guerres. La mise en place de la « Negro History Week » par Carter Woodson, les travaux d'Arthur Schomburg, de Joel Augustus Rogers ou encore les œuvres des écrivains Zora Neale Hurston et Langston Hughes participèrent ainsi activement à la popularisation de cette histoire.

Kendra Field revint finalement sur la période qui suivit le mouvement pour les droits civiques et le rapport particulier que celle-ci entretint avec l'histoire et la mémoire africaines-américaines, en mentionnant l'apport inestimable des travaux de l'historien Sterling Stuckey et notamment de son article pionnier intitulé « Through the Prism of Folklore " paru en 1968 et qui servit de socle théorique à son ouvrage Slave Culture : Nationalist Theory and the Foundations of Black America (1987). Les travaux de Sterling Stuckey n'étaient pas exempts de toute controverse à cause de leur teneur atypique et " radicale " au regard du courant africain-américain intégrationniste majoritaire des années 1950, pourtant leur contribution fut déterminante selon elle. Elle a noté que les recherches de Stuckey étaient parmi les premières à mettre l'accent sur l'importance du folklore (particulièrement les histoires transmises à l'oral) en tant qu'élément de connaissance privilégié de l'histoire de l'esclavage. En outre, le choc historiographique fut d'autant plus grand que lors de la publication de cet article en 1968, la conception académique de l'esclavage qui prédominait était encore celle initiée par Ulrich Phillips dans son ouvrage Life and Labor in the old South (1929): on y contestait catégoriquement l'authenticité des récits rédigés par les esclaves en raison de prétendues réécritures apocryphes de leurs œuvres par des abolitionnistes.

16 En 1956, la publication des travaux de l'historien Kenneth Stampp et du célèbre livre The Peculiar Institution instaurèrent une nouvelle dynamique dans les débats universitaires sur l'esclavage. Malgré l'importance de ces travaux qui relataient les tentatives de résistances des esclaves, aucun chercheur ne semblait envisager qu'il eût existé d'autres formes d'expressions culturelles au-delà du simple diptyque relationnel maitre-esclave selon Kendra Field. On ne vit finalement un changement définitif qu'à partir des années 1960 et 1970, lorsque la composante culturelle de l'histoire de l'esclavage fut mise en exergue par des chercheurs tels Sterling Stuckey ou encore Nell Irvin Painter. Dès lors et à l'aune des importantes avancées obtenues par le mouvement pour les droits civiques, il n'était plus possible de minorer la contribution du folklore et du storytelling africains-américains au sein de l'analyse historique de l'esclavage et de la Reconstruction. Kendra Field précisa également que ce renouveau notable s'était assorti d'une remise en question du mythe de «l'objectivité » par des historiens qui 
évoluaient constamment entre les sphères académiques et militantes, et dont ils furent simultanément « sujets et objets ».

En conclusion, Kendra Field évoqua l'intérêt contemporain grandissant pour les mémoires et histoires familiales africaines-américaines en dehors de l'université, et ce en raison de plusieurs facteurs comprenant la publication du roman Roots d'Alex Haley publié pour la première fois en 1976, son adaptation télévisuelle à succès l'année suivante et la "réémergence des réunions de familles africaines-américaines », en particulier celles financées par le National Council of Negro Women (une association nationale fondée en 1935 qui œuvre au bien-être des femmes et des familles noires). Les années 1970 et 1980 virent ainsi une curiosité relancée pour les histoires familiales africaines-américaines, que Kendra Field interprète comme une préfiguration de l'attrait actuel du grand public américain pour la "généalogie génétique ", comme en témoigne par exemple le succès irréfutable de l'émission "Finding Your Roots " présentée par l'historien africain-américain Henry Louis Gates Jr.

La conférence fut suivie de remarques par Hélène Le Dantec-Lowry et Hélène Quanquin, de la Sorbonne Nouvelle. Hélène Le Dantec-Lowry aborda les questions de méthodologie et d'historiographie utilisées par Kendra Field et les historiens africainsaméricains en général. Elle établit notamment un lien entre les sources utilisées et la notion " d'écriture de l'histoire depuis les marges » récemment exploré par le projet IDEX éponyme de SPC. Hélène Le Dantec-Lowry a ainsi évoqué la longue tradition d'écriture de l'histoire africaine-américaine par les « historiens amateurs » dont les ouvrages ont éclairé la compréhension de thématiques comme la participation des Africains-Américains pendant la Guerre d'Indépendance américaine ou la guerre de Sécession. Elle a également mis en parallèle le changement de paradigme concernant les sources sur l'esclavage opéré par Sterling Stuckey et l'historien Lawrence Levine dans son ouvrage Black Culture and Black Consciousness : Afro-American Folk Thought from Slavery to Freedom (1977). Elle a de plus souligné le rôle de la notion de classe - parfois reliée à la celle de la respectabilité évoquée par Kendra Field - dans l'historiographie africaine-américaine et la difficulté de mettre cette notion en application dans la grille d'analyse du sujet présenté lors de la conférence.

Hélène Quanquin a fait part quant à elle de son vif intérêt pour l'articulation entre histoire et militantisme, mais aussi envers les liens existant entre les historiens "amateurs " garants d'une histoire / mémoire familiale et les historiens «professionnels » qui participèrent d'une évolution de l'histoire en tant que discipline universitaire à part entière. Elle s'est montrée également intéressée par les enjeux de réconciliation qui sous-tendaient l'interprétation historique de la période de la Reconstruction et par le terme de " sources intimes " qui diffère des " sources personnelles » par son intrication au sein d'un environnement à la fois restreint mais aussi protégé. La question de la compilation des sources musicales et de l'appropriation potentielle par ses transcripteurs fut également évoquée avant d'effectuer un parallèle entre les silences des anciens esclaves et le mutisme traumatique de certains survivants de l'holocauste après la Seconde Guerre mondiale. 
INDEX

Thèmes : Actualité de la recherche

\section{AUTEUR}

PIERRE CRAS

Institut Catholique de Paris 